\title{
La investigación social a distancia en la enseñanza a distancia: La técnica DELPHI
}

\author{
(Distance research in distance education. An application of Delphi \\ technique) \\ Pablo Martín Pulido \\ SARA García CuEsta \\ Diana CALCERRADA GonZÁlez \\ (Dirección Adjunta de Investigación, IUED-UNED, España)
}

RESUMEN: La aplicación de las nuevas tecnologías de la información a la investigación social en el campo de la enseñanza a distancia presenta una serie de complejidades metodológicas por las especiales relaciones que se establecen, de un lado, entre investigadores y sujetos de estudio y, de otro lado, entre profesores, alumnos y gestores de la enseñanza a distancia. Los estudios sociológicos a distancia en educación a distancia conllevan esa doble dificultad, la que implica tener que llegar a individuos muy distantes geográficamente y la evanescencia de las relaciones en el sistema de la educación no presencial.

Nuevas tecnologías de la información, prospectiva, técnica Delphi, educación a distancia.

ABSTRACT: The application of new information technologies to the environment of distance education, in terms of social research, implies certain methodological complexities. The special relationships between the researcher and the subjects of study, together with the ones established among teachers, pupils and distance education managers, drive to redesign the methodological plannings. The sociological studies on distance education entail two difficulties: in one hand, subjects who are very distant geographically and on the other hand, the evanescence of interpersonal relationships that are a main aspect in the non-face-to-face education.

New information technologies, prospective, Delphi technique, distance learning. 
El presente artículo es fruto de una reflexión efectuada durante el periodo en que se viene realizando una investigación prospectiva sobre la educación a distancia, sus proyecciones futuras a nivel mundial en el contexto de la actual sociedad de la información ${ }^{1}$. El estudio trata de captar las transformaciones que está sufriendo el sistema de la enseñanza a distancia, principalmente a través de su vinculación con la implantación de nuevos sistemas de comunicación y relación entre las personas, así como de nuevos modelos de gestión de la información.

Se recogen, por un lado, las expectativas que abre este nuevo sistema de relaciones (profesor-alumno, burocracias universitarias, materiales didácticos y otros recursos, ...) en el marco de las transformaciones globales que se están produciendo en el mundo occidental en general. Por otro lado, se detecta también una cierta sensación de angustia ante los riesgos que se están asumiendo al modificar continuamente las bases sobre las que se ha asentado el sistema de aprendizaje durante los años que lleva recorridos la enseñanza a distancia. Nos cuenta Marshall Berman ${ }^{2}$ cómo la experiencia de la modernidad en cuanto que experiencia de un tiempo y un espacio es a la vez su propia contradicción, al desdibujar, desintegrar, renovar perpetuamente los contornos de lo que nos rodea. De igual manera, la enseñanza a distancia, de implantación relativamente reciente ${ }^{3}$, se está viendo obligada a asumir unos cambios de los cuales está por ver aún su efecto sobre el conjunto de los sistemas educativos universitarios. En concreto, la UNED tiene la responsabilidad de encauzar sus esfuerzos y tratar de incorporarse a estos procesos sin descuidar la atención a sus alumnos, en torno al potencial compensador de la institución (en este sentido, por encima de las incertidumbres de que hablamos), con el fin de llegar a los ciudadanos que, por diversos motivos, menos oportunidades tienen de acceder a la educación superior, máxime cuando este principio se encuentra en su texto fundacional.

La reflexión se centra, sobre todo, en los cambios ocurridos en la estructura organizativa de las propias instituciones, en consonancia con otras muchas transformaciones en distintos ámbitos. Como muestra de estas innovaciones, nos referimos concretamente a la mayor importancia depositada en las unidades de soporte informático o de «virtualización» de las relaciones entre las personas. Las nuevas posibilidades que ofrece el manejo informático de los datos en la gestión administrativa es un buen ejemplo de incorporación de las nuevas tecnologías a las instituciones educativas, y en esa misma línea deben ir, creemos, el resto de las adaptaciones.

\footnotetext{
${ }^{1}$ El estudio Proyecciones Futuras de la Enseñanza a Distancia en la Sociedad de la Información se enmarca dentro de la actividad de la Dirección Adjunta de Investigación del Instituto Universitario de Educación a Distancia de la UNED. En la actualidad no ha sido cerrado aún dicho estudio, lo que implica que los temas abordados en las páginas siguientes posiblemente encuentren eco en un segundo y definitivo artículo.

${ }^{2}$ Berman, M., Todo lo sólido se desvanece en el aire, SXXI, Madrid, 1988

${ }^{3}$ como sistema universitario «de peso», no hace siquiera 30 años que se generalizó en los países occidentales
} 
La investigación social a distancia en la enseñanza a distancia: La técnica DELPHI

Por lo tanto, una importante línea de trabajo desarrollada en este estudio se centra en la creciente complejidad de los medios de comunicación empleados en los procesos, tanto administrativos como de carácter docente o investigador, que existen en la UNED. En sus orígenes, eran «la radio, la televisión, las cintas magnetofónicas y videomagnéticas y cualquier otro medio análogo (...) correspondencia, reuniones periódicas en Centros Regionales y otros sistemas adecuados»" las vías de acceso de los estudiantes al material de estudio y a los docentes. En la actualidad, los desarrollos pedagógicos se sitúan en la línea del uso de Internet como herramienta de apoyo al estudio individual del alumno. "Hoy en día imperan los modelos de enseñanza-aprendizaje que recalcan la necesidad de incentivar el aprendizaje significativo y activo, donde el principal protagonista es el alumno. Internet se está convirtiendo en un medio adecuado para este fin, puesto que facilita la comunicación del profesor y alumno y de los alumnos entre sí, sin problemas de horarios ni distancias» ${ }^{5}$.

De estos argumentos de trabajo se extraerán unos marcos de referencia para la toma de decisiones que sirvan de apoyo, en lo que respecta a los sistemas gestión de la enseñanza a distancia, a instituciones de educación a distancia como la UNED.

Precisamente, el propósito de estas líneas no se orienta a presentar las conclusiones del estudio, todavía por delimitar. La propuesta de este artículo gira en torno a las cuestiones metodológicas que implica la investigación sobre educación a distancia -por las características peculiares que la definen- y, ligado a esto, la puesta en práctica desde la propia Universidad a distancia de una técnica de investigación social tan peculiar como es la técnica Delphi. Empezaremos con esto segundo.

A grandes rasgos, la técnica Delphi se define como la sucesión de recogidas y devoluciones de información de y a expertos en el tema a estudiar (en este caso específico, expertos en diferentes aspectos del sistema de enseñanza a distancia), de tal manera que el resultado es una serie de protocolos de actuación («la previsión no tiene un carácter final, sino instrumental, al servicio de la planificación y decisión $»^{\circ}$ ), un consenso de los expertos en torno al tema estudiado, en la medida en que se va procesando la información aportada?

\footnotetext{
${ }^{4}$ García Aretio, L. y Almenar Ibarra, N., 25 años de la UNED en números, UNED, Madrid, 1999, en referencia al Decreto 2310/72.

${ }^{5}$ González Boticario J. y Gaudioso, E., Introducción a la enseñanza en Internet, UNED (documentación interna)

${ }^{6}$ Landeta, J., El método Delphi, Ariel, Barcelona, 1999

${ }^{7}$ La definición que ofrece Landeta es la siguiente:

Método de estructuración de un proceso de comunicación grupal que es efectivo a la hora de permitir a un grupo de individuos, como un todo, tratar un problema complejo, es decir, obtener una opinión grupal fidedigna a partir de un grupo de expertos.
} 
Pablo Martín Pulido, Sara García Cuesta, Diana Calcerrada González

En un comentario más detallado, las premisas que debe, entonces, cumplir esta técnica son:

D Basarse en un proceso iterativo, en que los expertos reciben y devuelven sucesivamente la información, cada vez más elaborada, a los miembros del equipo investigador.

D Anonimato que debe respetarse en la medida de lo posible para evitar implícitos entre los expertos del grupo (con la consiguiente pérdida de información) y previsión de respuestas a los comentarios de los otros expertos.

Detroalimentación controlada de la información. Ésta debe ser la única que se manifieste a los expertos (evitando las comunicaciones entre ellos) y de carácter general, ofreciendo sugerencias, o informaciones adicionales más que opiniones concretas.

D Respuesta estadística de grupo, en cuanto que los datos sean cuantificables, para poder dar una medida de grupo (principalmente se buscan las tendencias centrales y las dispersiones).

\section{DIAGRAMA MÉTODO DELPHI}

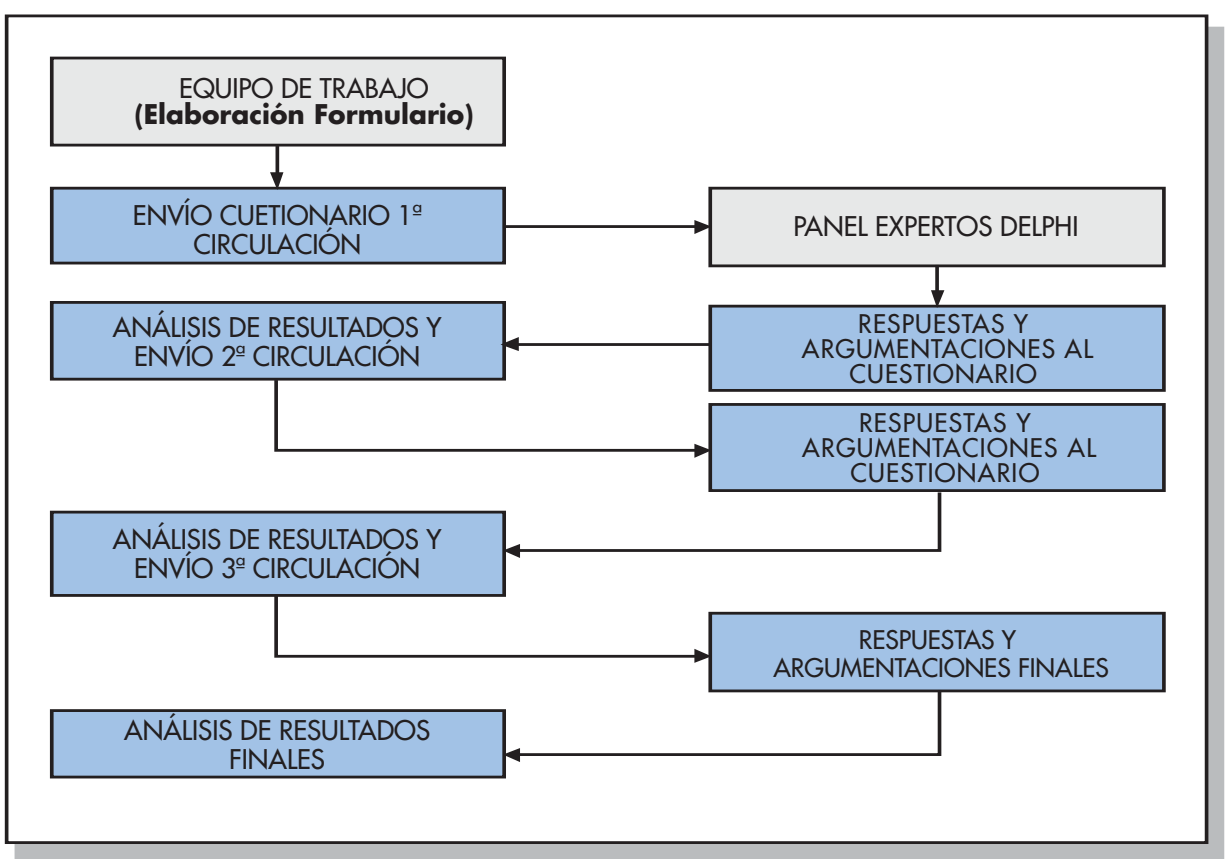


Por pasos, en primer lugar se elabora el guión de trabajo, a modo de cuestionario abierto, que nos provea de temática suficiente como para abarcar las diferentes dimensiones del objeto de estudio en un estadio más avanzado de la investigación. En nuestro caso, estamos dirigidos por Javier Callejo, profesor titular en la Facultad de Sociología de la UNED y Director Adjunto de Investigación del IUED. Él fue quien diseñó las baterías de temas a tratar, que, a grandes rasgos, son las siguientes:

- tendencias generales actuales en la enseñanza a distancia

- bases sociales implicadas

- relación entre enseñanza a distancia y presencial

- relación con el sistema productivo

- tamaño de las instituciones

- financiación

- recursos personales

- enseñanza a distancia y globalización

- metodologías de enseñanza

- relación profesor - alumno.

El hecho de delimitar los temas ya plantea un problema de «parcelación de la realidad», que se ha intentado contrarrestar con la suficiente libertad (inicial) que se ofrece a los entrevistados para explicitar ellos mismos otras cuestiones de interés ${ }^{8}$. De esta manera, en la primera ola del estudio se recogen datos «lo más cualitativamente posible ${ }^{9} \mathrm{y}$ se les dota de una homogeneidad y consistencia interna para la primera devolución. He aquí la tarea más importante a que se enfrentan los investigadores. Tomaremos el trabajo de los entrevistados-expertos de tal manera que se puedan definir las líneas discursivas que encierra este tema de estudio. Ahí reside el valor de la información aportada, del carácter de expertos de los individuos seleccionados para la investigación. Su conocimiento explícito de los conceptos manejados, las teorías, las opiniones que circulan en estos específicos contextos es lo que verdaderamente nos interesa destacar. Posteriormen-

\footnotetext{
${ }^{8}$ Queda expuesto así el eterno dilema entre las técnicas cuantitativas y cualitativas: más cerradas unas en torno a la supuesta omnisciencia y objetividad del investigador (enfatizan la búsqueda), más dispuestas a la autorreferencia por parte de los sujetos investigados, otras (enfatizan el encuentro).

${ }^{9}$ Con esta cuestión, compleja de entender a simple vista, no queremos más que una recogida de ideas, impresiones, que posteriormente son agrupadas temáticamente, desarrollando unos primeros hilos discursivos, que se completarán y matizarán en sucesivas olas del cuestionario. Esto no puede considerarse un análisis de contenido de corte cualitativo, es simplemente un «recuento» de opiniones que servirán de base, en la línea de desarrollo teórico de la técnica Delphi, a ulteriores cuestionarios.
} 
te, las conclusiones consisten en una reelaboración de esa misma información, dándoles coherencia en el sentido de que sea una explicación del fenómeno como hecho social. Esto es, consecutivamente se perfilan y destacan las grandes líneas en que se está avanzando en el campo de la enseñanza a distancia.

La selección de la base muestral planteó de la misma manera una serie de problemas que especificamos a continuación: primero, el hecho de abordar dimensiones de tal calado sobre la educación a distancia implicó complejidades en la definición de perfiles para los entrevistados. Las decisiones metodológicas en lo que respecta a la definición de los perfiles fueron salvadas por dos vías:

- aumentando la propia muestra, de tal manera que en la «selección natural» (mortandad de la muestra) no se descolgara un contingente demasiado amplio que no permitiera un análisis consistente. La muestra se amplió finalmente a 300 individuos.

- Seleccionando sujetos con un conocimiento lo suficientemente amplio sobre los diversos aspectos de la enseñanza a distancia de modo que puedan responder a todos los aspectos recogidos en el cuestionario. Así, las respuestas parciales quedaban excluidas.

Un segundo problema que se nos planteó fue el de seleccionar los individuos o instituciones por su pertinencia para el estudio. Esto es, la decisión sobre la selección implica un profundo conocimiento de las instituciones que se dedican a la enseñanza a distancia, con el agravante de que es un estudio a nivel mundial. El rastreo que efectuamos también estuvo condicionado, como se explicará a continuación, por el medio de comunicación empleado a lo largo del estudio, que no es otro que Internet. Esto, unido al difícil acceso a las bases de datos, listados, who-is-whos,..., que, además de ser reducidos -cada institución suele elaborar únicamente su propio listado-, son escasos, hizo que la fase de preparación de la muestra se convirtiera en una auténtica obra de artesanía ${ }^{10}$.

Realizamos una exhaustiva búsqueda por las instituciones públicas y privadas dedicadas a la enseñanza, presencial y a distancia, con el fin de entresacar de ellas las direcciones de correo electrónico para contactar con los expertos. La necesidad de encontrar el correo electrónico clave que nos permitiera localizar a la persona en cuestión también nos procuró unas cuantas preocupaciones. Aun así, el hecho de tener una dirección de e-mail no nos aseguraba la respuesta, dado que muchos de los seleccionados eran altos y ocupados responsables de sistemas educativos. Por otra parte, eso era lo que se buscaba.

\footnotetext{
${ }^{10}$ Se introdujeron, además, cuotas en la selección de los sujetos dependiendo de su vinculación con el sistema educativo, altos responsables del sistema, responsables de instituciones de educación a distancia y presencial, ... con el fin de abarcar, merced a las distintas posiciones e intereses dentro del sistema educativo, una mayor variedad de opiniones.
} 
La investigación social a distancia en la enseñanza a distancia: La técnica DELPHI

Una última cuestión que se planteó referida a la base de datos, fue la de crear grupos de trabajo institucional, esto es, organizar grupos de expertos que funcionaran como un solo sujeto, con una única voz, pero que fuera fruto de debates en el seno de la propia institución de origen. De tal manera, hubiéramos podido suplir la carencia (o disponibilidad) de esos «grandes expertos» que tanto costó localizar y hubiéramos tenido líneas de decisión institucionales (se entiende también que insertas en la política educativa de cada institución, que en definitiva son las que dirigen el cambio en las estructuras del sistema). Si bien esto nos pareció en un primer momento acertado, finalmente se desestimó por suponer problemas adicionales en cuanto a la configuración de los grupos de trabajo: ¿cuántas personas los compondrían?, ¿quién elegiría a los componentes?, ¿qué representatividad tendría su composición?, ¿cómo se controlaría el debate?, ¿serían multidisciplinares realmente?, ...

Como se ha comentado anteriormente, en todo momento, la herramienta utilizada tanto en la elaboración de la base de datos, en la contactación y en las sucesivas comunicaciones con los entrevistados, fue la informática. La necesidad de llegar a todos los rincones del mundo sencilla y rápidamente y con el menor costo nos obligó al uso del correo electrónico y a la elaboración de una página web donde exponer la información relevante del estudio, así como los resultados que se fueran obteniendo ${ }^{11}$. La experiencia resultó muy positiva por las ventajas que a simple vista ofrece esta herramienta: rapidez en la transmisión de la información, visualización inmediata de la información a través de la página web de la UNED ${ }^{12}$, posibilidades de tratamiento informático de la información, ... ${ }^{13}$. Se pretendía, además, ahondar en el debate en torno al uso de las nuevas tecnologías de la información ${ }^{14}$, evidenciar sus potencialidades en el sistema de la enseñanza a distancia (en su faceta de gestión del conocimiento) y en la concreta aplicación a los estudios sociales.

\footnotetext{
${ }^{11}$ Se puede visitar la página web desarrollada para la investigación en la dirección: http://www.iued.uned.es/iued/investigacion/delphi/index.html

${ }^{12}$ De vital importancia es la vinculación del estudio a la UNED como institución, por su poder simbólico en este ámbito.

${ }^{13} \mathrm{El}$ hecho de que se innove en los procesos para resolver los problemas indica que las Tecnologías de la Información no evolucionan para sí mismas (al contrario de lo que opina McLuhan, a veces, el medio no es el fin), sino que modernizan y diluyen los sistemas jerárquicos, el autoritarismo, las relaciones de subordinación y obediencia y el acceso restringido a la información, entendidos estos fenómenos como consustanciales a la burocracia de las sociedades occidentales. Los grupos de trabajo a través de internet, comunicación sincrónica y diacrónica, la informatización de archivos y grandes bases de datos y su manejo descentralizado son proyectos, en otros casos ya realizaciones, que abordan el hecho de la democratización en el acceso a la información. Esta idea está tomada de Ann Petrides, Lisa (comp.): Case studies on information technology in higher education: implications for policy and practice, Idea Group Publishing, 2000

${ }^{14}$ La bibliografía sobre este tema es abundante (y crece exponencialmente). Un curioso ejemplo es la reflexión de Pierre Levy sobre la virtualización como una fase más del proceso de desarrollo cultural (Lévy, Pierre, ¿Qué es lo virtual?, Paidos, Barcelona, 1999)
} 
En primer lugar, la página web se ideó para que fuera, además de una herramienta para la comunicación, un «escaparate» del estudio: una guía completa de introducción tanto a las bases del proyecto, sus objetivos y tareas, como a la metodología explicada paso a paso, sus fases, elementos intervinientes. Esto último con el fin de contextualizar la importancia vital que se da en la investigación al trabajo de los expertos. En definitiva, la página está destinada, fundamentalmente, a facilitar la comunicación con el panel de expertos durante el desarrollo del estudio. Aporta, además, credibilidad al estudio y es un factor e motivación destinado a los expertos seleccionados. Por último, como se comentará a continuación, el sitio web fue diseñado como una importante herramienta que aporta flexibilidad al estudio al permitir la centralización y ágil recogida de información y la rápida exposición pública de los datos.

En ella se pueden encontrar los objetivos del estudio, la metodología y el plan de trabajo, así como la presentación del equipo investigador. La imagen muestra la página de entrada, con los distintos apartados que recogen la información necesaria tanto para los expertos participantes, como para cualquier persona que tenga interés en acceder a la teoría y la práctica de la técnica Delphi.

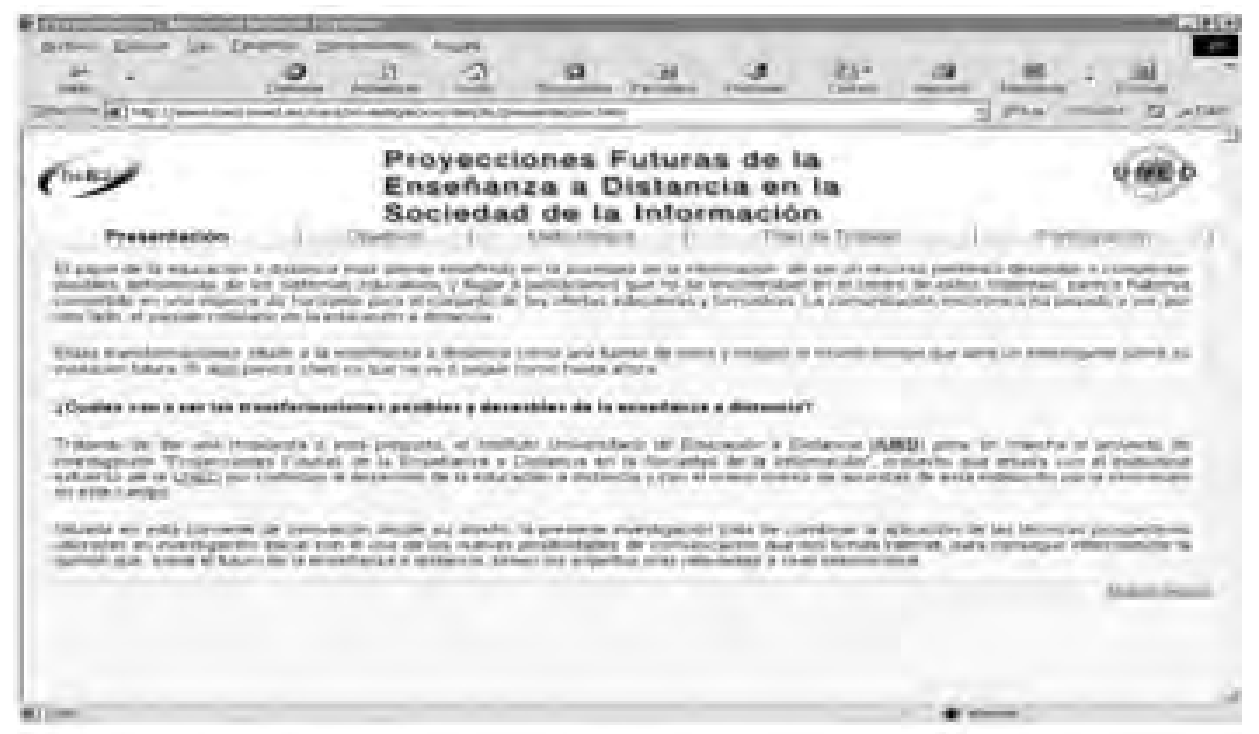

En segundo lugar, se planteó la utilización de la plataforma aLF, que fue desarrollada en su día por la Unidad Técnica de Investigación y Formación en Recursos Tecnológicos (TecInfor) de la UNED. Esta herramienta permite disponer de espacio para áreas de trabajo personales, áreas de almacenamiento de información, comunicación a través de foros, publicación de noticias, etc ... De esta manera, se crearía un grupo de trabajo (manteniendo el anonimato), cuyo funcionamiento vendría determinado por la colaboración de los expertos en el apor- 
La investigación social a distancia en la enseñanza a distancia: La técnica DELPHI

te de la información, y la administración del grupo y reelaboración de dicha información por parte del equipo investigador. Para nuestro fin, nos interesaron, principalmente, las oportunidades que ofrece el hecho del manejo de los datos y las condiciones de envío y recepción de los resultados.

Inicialmente, aLF no fue diseñada para estos fines, pero se han podido aprovechar las ventajas antes mencionadas ya que un espacio virtual como el de esta plataforma evita el procesamiento «manual» de una cantidad tan grande de datos como la que se creará una vez se pongan en marcha las sucesivas oleadas del estudio. Por tanto, el diseño del estudio había de tener en cuenta un dispositivo que simplificara el proceso de creación de las bases de datos y su operatividad. Explorando las posibilidades de la plataforma, pudimos confeccionar, a grandes rasgos, la siguiente lista de ventajas e inconvenientes, que finalmente dio como resultado una solución mixta: se decidió incorporar algunas utilidades de la plataforma, aunque, por motivos diversos, se mantuvieron mecanismos «manuales» de trabajo.

Ventajas:

- la plataforma aLF permite realizar los envíos masivos de cuestionarios de la primera ola, así como las sucesivas devoluciones de la información se realizarían de manera automática sin necesidad de mandar los e-mails uno por uno.

- permite, asimismo, la grabación automática de los datos, ya que los expertos no remitirían la información, ni siquiera vía e-mail, sino que accederían directamente a la plataforma y allí cumplimentarían el cuestionario, con lo que evitarían la tediosa fase de grabación de datos.

- se pueden almacenar una gran cantidad de datos, una vez que se reciba la información de la primera oleada y, posteriormente, explotar las bases creadas, convertibles, por ejemplo, a formato .sav para el tratamiento con el paquete estadístico SPSS.

- de esta manera, se reducen las labores que implica la comunicación con los expertos, el envío y recepción de la información y cuestionarios, informes de las oleadas, etc ..., ya que podrían acceder a ellos a través de la misma página creada para la investigación.

Inconvenientes:

- el principal inconveniente no procedió del diseño de la plataforma, sino que fue fruto de la difícil coordinación entre el equipo investigador y el equipo de mantenimiento de la plataforma. La inseguridad de los investigadores a la hora de movernos por la plataforma, junto a los constantes interrogantes que planteaba el diseño del estudio, hizo indispensable la ayuda técnica en todo momento.

- el punto anterior tiene eco en la premura con que se nos presentan los plazos de cada fase. Un estudio de esta magnitud debe respetar los plazos, so- 
Pablo Martín Pulido, Sara García Cuesta, Diana Calcerrada González

bre todo en sus fases iniciales, para no acumular retrasos de tiempo en estadios más avanzados. Por tanto, ante estos problemas de coordinación, se tomó la decisión de utilizar la plataforma para procedimientos indispensables en el desarrollo del estudio, a pesar de las potenciales ayudas que ofrecía su uso en etapas posteriores.

- por otra parte, uno de los pocos problemas (y no por ello despreciable) que planteaba el uso de aLF en esta investigación fue el hecho de que el formato de cuestionario (formulario de pregunta-respuesta prediseñado) que tiene configurada la plataforma está en español, cuando es de vital importancia la distribución y tratamiento de un cuestionario en inglés en un estudio a escala internacional, como ocurre en este caso.

Por todos los factores anteriormente expuestos, se resolvió enviar manualmente vía e-mail una carta de presentación a los expertos, exponiendo la finalidad del estudio y solicitando su participación. En la misma se incluirá un enlace al sitio web de la investigación, desde la que se podrá descargar el cuestionario, para posteriormente remitirlo de la misma manera al equipo investigador. La página está alojada en la plataforma, donde se irán archivando, según se avance en la investigación, los sucesivos informes de recapitulación, nuevas convocatorias, etc...

\section{CONCLUSIONES}

La intención de estas líneas es mostrar las posibilidades que ofrecen las nuevas tecnologías de la comunicación en la investigación social aplicada. La rapidez en la comunicación a larga distancia y la simplificación en los procesos de contactación con los entrevistados en este caso son valores a tener en cuenta, ya que eliminan barreras que hasta ahora eran casi infranqueables.

Como se ha reseñado más arriba, estamos aún en el camino de la incorporación al estudio de todas las potencialidades que ofrece el uso de la plataforma aLF, con menos premura, tal vez, de la deseada, debido a las dificultades que nos hemos encontrado en la realización de la investigación. Todo ello no ha evitado que en el diseño del proyecto recogiéramos aquellos procesos para los cuales el uso de la plataforma es imprescindible.

No obstante, quienes en la actualidad estamos vinculados a este proyecto creemos que aún queda mucho por hacer en cuanto a reducir estas barreras que se acaban de mencionar. La consolidación de las herramientas que en la actualidad están en fase experimental es un paso definitivo para hacer de las nuevas tecnologías, las fieles compañeras en el proceso de la investigación sociológica. Sin quitar por ello un ápice de responsabilidad a los profesionales de la Sociología en cuanto a la formación en, y manejo de, las nuevas tecnologías en aquellos aspectos de la práctica del oficio que lo requieren. 
La investigación social a distancia en la enseñanza a distancia: La técnica DELPHI

\section{REFERENCIAS BIBLIOGRÁFICAS}

AnN Petrides, Lisa (comp.). (2000). Case studies on information technology in higher education: implications for policy and practice, Idea Group Publishing.

Berman, M. (1988). Todo lo sólido se desvanece en el aire. Madrid: Siglo XXI.

García Aretio, L. Y Almenar IbarRa, N. (1999). 25 años de la UNED en números. Madrid: UNED.

García Boticario, J., Gaudioso, E., Introducción a la enseñanza en Internet, UNED (documentación interna)

Hanna, D. (2000) Higher education in an era of digital competition. Atwood Publishing: Madison (Wisconsin).

Landeta, J. (1999) El método Delphi. Barcelona: Ariel.

LÉVY, PIERRE. (1999) ¿Qué es lo virtual? Barcelona: Paidos.

McLuhan, M. (1998). La Galaxia Gutemberg. Barcelona: Círculo de Lectores.

\section{PERFIL ACADÉMICO Y PROFESIONAL DE LOS AUTORES}

Pablo Martín Pulido y Sara García Cuesta son sociólogos, miembros del equipo de la Dirección Adjunta de Investigación del Instituto Universitario de Educación a Distancia (IUED) de la UNED.

Diana Calcerrada González es socióloga, actualmente trabaja en una empresa de investigación de mercados.

Pablo Martín Pulido: pblmartin@bec.uned.es

Sara García Cuesta: sgc@bec.uned.es 